\title{
THE ALIGNMENT OF THE POLARIZATION OF HERBIG Ae/Be STARS WITH THE INTERSTELLAR MAGNETIC FIELD*
}

\author{
Cláudia V. Rodrigues ${ }^{1}$, Marília J. Sartori ${ }^{2}$, Jane Gregorio-Hetem ${ }^{3}$, and A. Mário Magalhães ${ }^{3}$ \\ ${ }^{1}$ Instituto Nacional de Pesquisas Espaciais/MCT, Av. dos Astronautas, 1758, 12227-010, São José dos Campos, SP, Brazil; claudiavr@ das.inpe.br \\ ${ }^{2}$ Laboratório Nacional de Astrofsica/MCT, 37504-364, Itajubá, MG, Brazil \\ ${ }^{3}$ Instituto de Astronomia, Geofísica e Ciências Atmosféricas/Un. São Paulo, R. do Matão, 1225, 05508-900, São Paulo, SP, Brazil \\ Received 2009 March 17; accepted 2009 April 23; published 2009 June 5
}

\begin{abstract}
We present a study of the correlation between the direction of the symmetry axis of the circumstellar material around intermediate mass young stellar objects and that of the interstellar magnetic field. We use CCD polarimetric data on 100 Herbig Ae/Be stars. A large number of them show intrinsic polarization, which indicates that their circumstellar envelopes are not spherical. The interstellar magnetic field direction is estimated from the polarization of field stars. There is an alignment between the position angle of the Herbig Ae/Be star polarization and that of the field stars for the most polarized objects. This may be an evidence that the ambient interstellar magnetic field plays a role in shaping the circumstellar material around young stars of intermediate mass and/or in defining their angular momentum axis.
\end{abstract}

Key words: stars: pre-main sequence - ISM: magnetic fields - polarization

Online-only material: machine-readable table

\section{INTRODUCTION}

The role of magnetic field in star formation processes is a longstanding problem in astrophysics. Magnetic field is proposed by some authors (e.g., Mouschovias \& Ciolek 1999) to act in support of molecular clouds against the gravitational force. A different view is that these clouds are not stable and exist as ephemeral structures. In this case, the turbulence drives the interstellar medium (ISM) large-scale structure (e.g., McKee \& Ostriker 2007). It is possible to address this issue by the search of evidences about the importance of the interstellar magnetic field in the star-forming process. From an observational point of view, many works have searched for a correlation between the direction of the interstellar magnetic field and the geometry of young stellar objects (YSOs) traced by the disk axis, outflow direction, or observed polarization. We cite two of them. Tamura \& Sato (1989) have found a correlation between the interstellar magnetic field direction and the infrared polarization angle in a sample of 47 YSOs in the Taurus-Auriga molecular cloud. This sample is dominated by T Tauri stars. Recently, Ménard \& Duchêne (2004) have studied 37 T Tauri stars in the same region and found no correlation between the local magnetic field and the geometry of the YSOs.

Herbig Ae/Be objects (HAeBe) are pre-main-sequence stars, analogue to $\mathrm{T}$ Tauri stars, but of intermediate mass. Lists of HAeBe stars can be found in the compilation of The et al. (1994) and in the Pico dos Dias Survey (PDS), a search for T Tauri stars based on the Infrared Astronomical Satellite (IRAS) colors (Gregorio-Hetem et al. 1992; Torres et al. 1995). In spite of the focus on low-mass YSOs, the PDS has also found around a 100 HAeBe candidates (Vieira et al. 2003; Torres 1999). In this work, we revisit the issue of alignment of the interstellar magnetic field with the YSO geometry using a large sample of HAeBe objects. The study of the polarization in the context of the circumstellar material properties will be done elsewhere (M. J. Sartori et al. 2009, in preparation). In Section 2,

\footnotetext{
* Based on observations made at the Observatório do Pico dos Dias, Brazil, operated by the Laboratório Nacional de Astrofísica.
}

we describe the acquisition and reduction of the polarimetric data and the technique to calculate the interstellar and intrinsic stellar polarization. The results and discussion are presented in Section 3. In the last section, we summarize our findings.

\section{OBSERVATIONS}

We obtained polarimetric data on 102 fields containing probable HAeBe stars selected from Thé et al. (1994), Torres (1999), and Vieira et al. (2003). The observations were done with the $0.60 \mathrm{~m}$ Boller \& Chivens telescope at the Observatório do Pico dos Dias, Brazil, operated by the Laboratório Nacional de Astrofísica, Brazil from 1998 to 2002. We used a CCD camera modified by the polarimetric module described in Magalhães et al. (1996). The used detector is an SITe back-illuminated $\mathrm{CCD}, 1024 \times 1024$ pixels. This combination of telescope and instrumentation results in a field of view of $10.5 \times 10.5$ (1 pixel $=0.62)$. The data were taken using a $V$ filter. We have collected eight images of each field. Table 1 lists the observation date and the integration time (for one image) for each field. The reduction followed the standard steps of differential photometry using the IRAF facility ${ }^{4}$ and the package PCCDPACK (Pereyra 2000; Pereyra \& Magalhães 2002). Polarized standard stars were observed to convert the instrumental position angle to the equatorial reference frame. Unpolarized standard star measurements were consistent with zero within the errors and hence no corrections for instrumental polarization were applied to the data. Measurements using a Glan filter, which provide the efficiency of the instrument, indicate that no correction is needed considering the instrumental precision. The observed polarization data are presented in Table 1 . It contains the 102 program stars plus two confirmed post-AGB that contaminate the PDS sample and are not included in the analysis.

The observed polarization of a YSO is usually composed of two components: an intrinsic polarization plus an interstellar polarization component. The intrinsic component is produced by

\footnotetext{
4 IRAF is distributed by National Optical Astronomy Observatories, which is operated by the Association of Universities for Research in Astronomy, Inc., under contract with the National Science Foundation.
} 
Table 1

Polarimetry of HAeBe Objects

\begin{tabular}{|c|c|c|c|c|c|c|c|c|c|c|c|c|c|c|}
\hline \multirow[t]{2}{*}{ Object } & \multirow{2}{*}{$\begin{array}{c}V^{\mathrm{a}} \\
(\mathrm{mag})\end{array}$} & \multirow{2}{*}{$\begin{array}{c}\text { Exposure } \\
\text { (s) }\end{array}$} & \multirow[t]{2}{*}{ UT Date } & \multicolumn{3}{|c|}{ Observed } & \multicolumn{4}{|c|}{ Foreground } & \multicolumn{3}{|c|}{ Intrinsic } & \multirow[t]{2}{*}{ Ref. $^{b}$} \\
\hline & & & & $\begin{array}{c}P \\
(\%)\end{array}$ & $\begin{array}{l}\sigma_{P} \\
(\%)\end{array}$ & $\begin{array}{l}\text { P.A. } \\
\text { (deg) }\end{array}$ & $\begin{array}{c}\text { No. of } \\
\text { Objects }\end{array}$ & $\begin{array}{c}P \\
(\%) \\
\end{array}$ & $\begin{array}{l}\sigma_{P} \\
(\%)\end{array}$ & $\begin{array}{l}\text { P.A. } \\
\text { (deg) }\end{array}$ & $\begin{array}{c}P \\
(\%)\end{array}$ & $\begin{array}{l}\sigma_{P} \\
(\%)\end{array}$ & $\begin{array}{l}\text { P.A. } \\
\text { (deg) }\end{array}$ & \\
\hline PDS 002 & 10.9 & 100 & 1998 Nov 25 & 0.128 & 0.045 & 135.4 & 12 & 0.190 & 0.022 & 107.0 & 0.161 & 0.050 & 176.1 & 1 \\
\hline PDS 004 & 10.7 & 120 & 1998 Nov 25 & 1.018 & 0.033 & 45.8 & 48 & 0.557 & 0.020 & 49.8 & 0.473 & 0.039 & 41.0 & 1 \\
\hline HD $23302^{c}$ & 3.7 & 5 & 1999 Jul 28 & 0.238 & 0.038 & 113.8 & 0 & $\ldots$ & $\ldots$ & $\cdots$ & $\ldots$ & $\ldots$ & $\ldots$ & 3 \\
\hline HD $23480^{c}$ & 4.2 & 7 & 1999 Jul 28 & 0.507 & 0.033 & 141.2 & 0 & $\cdots$ & $\cdots$ & $\cdots$ & $\ldots$ & $\cdots$ & $\cdots$ & 3 \\
\hline PDS 168 & 17.3 & 300 & 1999 Jul 29 & 8.377 & 0.278 & 62.0 & 33 & 2.544 & 0.015 & 62.9 & 5.835 & 0.278 & 61.6 & 1 \\
\hline PDS 172 & 7.6 & 30 & 1998 Nov 24 & 0.354 & 0.013 & 52.1 & 30 & 0.126 & 0.070 & 127.8 & 0.469 & 0.071 & 48.4 & 1,2 \\
\hline PDS 176 & 9.8 & 100 & 1998 Nov 24 & 0.141 & 0.021 & 64.4 & 33 & 0.173 & 0.019 & 17.6 & 0.230 & 0.028 & 88.7 & 1,2 \\
\hline PDS 110 & 10.4 & 200 & 1998 Nov 24 & 0.366 & 0.066 & 27.6 & 21 & 0.367 & 0.024 & 117.9 & 0.733 & 0.070 & 27.7 & 3 \\
\hline PDS 178 & 7.8 & 25 & 1998 Nov 24 & 0.120 & 0.021 & 140.6 & 14 & 2.369 & 0.094 & 83.3 & 2.421 & 0.096 & 172.0 & 1 \\
\hline PDS 179 & 9.7 & 90 & 1998 Nov 25 & 0.336 & 0.027 & 80.0 & 61 & 0.260 & 0.015 & 87.8 & 0.111 & 0.031 & 60.3 & 1 \\
\hline PDS 180 & 10.1 & 120 & 1999 Apr 8 & 0.266 & 0.026 & 58.9 & 25 & 0.188 & 0.011 & 90.6 & 0.248 & 0.028 & 37.5 & 1,2 \\
\hline PDS 114 & 10.0 & 90 & 1998 Nov 25 & 0.161 & 0.032 & 35.5 & 89 & 0.060 & 0.022 & 10.5 & 0.131 & 0.039 & 45.7 & 1 \\
\hline PDS 184 & 10.1 & 120 & 1999 Apr 8 & 0.421 & 0.017 & 118.5 & 65 & 0.616 & 0.013 & 122.4 & 0.207 & 0.021 & 40.4 & 1 \\
\hline PDS 183 & 8.3 & 30 & 1998 Nov 24 & 0.368 & 0.011 & 41.9 & 21 & 1.849 & 0.084 & 88.1 & 1.900 & 0.085 & 3.6 & 1,2 \\
\hline PDS 185 & 6.5 & 2 & 2002 Oct 19 & 0.455 & 0.043 & 179.0 & 22 & 0.972 & 0.099 & 168.8 & 0.568 & 0.108 & 70.7 & 1 \\
\hline PDS 190 & 9.3 & 90 & 1998 Nov 25 & 0.390 & 0.149 & 75.5 & 20 & 0.319 & 0.071 & 104.1 & 0.345 & 0.165 & 50.0 & 1 \\
\hline PDS 191 & 8.9 & 40 & 1999 Apr 8 & 0.173 & 0.007 & 75.4 & 5 & 0.153 & 0.011 & 41.4 & 0.183 & 0.013 & 100.8 & 1,2 \\
\hline PDS 192 & 9.9 & 40 & 2002 Oct 19 & 0.359 & 0.026 & 46.8 & 93 & 0.081 & 0.033 & 18.1 & 0.323 & 0.042 & 52.9 & 1 \\
\hline PDS 193 & 13.9 & 400 & 1998 Nov 25 & 2.183 & 0.114 & 86.3 & 9 & 0.087 & 0.057 & 17.2 & 2.249 & 0.127 & 87.0 & 1,2 \\
\hline PDS 194 & 9.8 & 100 & 1999 Apr 8 & 0.300 & 0.023 & 31.1 & 6 & 2.035 & 0.304 & 123.9 & 2.334 & 0.305 & 33.5 & 1,2 \\
\hline PDS 016 & 9.0 & 40 & 1998 Nov 24 & 0.269 & 0.036 & 12.3 & 29 & 0.227 & 0.033 & 176.4 & 0.142 & 0.049 & 41.0 & 1 \\
\hline PDS 201 & 8.9 & 50 & 1998 Nov 24 & 0.573 & 0.037 & 58.4 & 41 & 0.202 & 0.011 & 35.0 & 0.459 & 0.039 & 67.7 & 1 \\
\hline PDS 019 & 13.9 & 200 & 1999 Apr 8 & 0.274 & 0.033 & 129.0 & 37 & 0.259 & 0.013 & 109.9 & 0.175 & 0.035 & 162.1 & 1 \\
\hline PDS 020 & 10.6 & 90 & 1998 Nov 25 & 0.571 & 0.032 & 165.7 & 146 & 1.171 & 0.011 & 161.1 & 0.614 & 0.034 & 66.8 & 1 \\
\hline PDS 021 & 10.4 & 100 & 1998 Nov 25 & 1.598 & 0.022 & 29.7 & 41 & 0.738 & 0.013 & 32.6 & 0.867 & 0.026 & 27.2 & 1 \\
\hline PDS 022 & 10.2 & 100 & 1998 Nov 25 & 0.201 & 0.018 & 102.0 & 82 & 0.083 & 0.010 & 134.6 & 0.182 & 0.021 & 89.8 & 1 \\
\hline PDS 225 & 6.9 & 2 & 1999 Apr 11 & 0.974 & 0.044 & 26.3 & 15 & 0.681 & 0.219 & 172.7 & 0.948 & 0.223 & 47.0 & 1 \\
\hline HD 51585 & 11.1 & 80 & 1999 Apr 11 & 0.522 & 0.045 & 43.3 & 207 & 0.183 & 0.018 & 12.5 & 0.464 & 0.048 & 53.5 & 3 \\
\hline PDS 241 & 12.1 & 120 & 1999 Apr 11 & 3.672 & 0.037 & 104.8 & 315 & 0.324 & 0.009 & 127.4 & 3.451 & 0.038 & 102.9 & 1 \\
\hline PDS 249 & 14.2 & 300 & 1999 Apr 8 & 1.703 & 0.190 & 54.6 & 204 & 0.545 & 0.007 & 147.1 & 2.246 & 0.190 & 55.2 & 1 \\
\hline PDS 272 & 9.8 & 40 & 1999 Jan 22 & 0.130 & 0.026 & 109.6 & 129 & 0.183 & 0.019 & 83.1 & 0.147 & 0.032 & 150.7 & 1 \\
\hline PDS 277 & 10.0 & 45 & 1999 Jan 22 & 0.040 & 0.049 & 97.0 & 178 & 0.537 & 0.014 & 51.2 & 0.540 & 0.051 & 139.0 & 1 \\
\hline PDS 031 & 8.5 & 8 & 1999 Apr 11 & 0.138 & 0.043 & 82.1 & 77 & 0.687 & 0.035 & 87.8 & 0.552 & 0.055 & 179.2 & 1 \\
\hline HBC 563 & 14.2 & 200 & 1999 Jan 22 & 3.307 & 0.241 & 177.7 & 54 & 0.067 & 0.021 & 26.6 & 3.272 & 0.242 & 177.2 & 2 \\
\hline PDS 033 & 12.3 & 80 & 1999 Jan 22 & 0.862 & 0.071 & 76.7 & 82 & 0.034 & 0.025 & 121.1 & 0.862 & 0.075 & 75.5 & 1 \\
\hline PDS 034 & 14.0 & 400 & 1999 Jan 23 & 2.799 & 0.418 & 117.6 & 229 & 0.515 & 0.006 & 104.9 & 2.344 & 0.418 & 120.3 & 1 \\
\hline HD 76534 & 8.0 & 10 & 1999 Apr 11 & 0.466 & 0.017 & 127.4 & 29 & 2.813 & 0.041 & 76.3 & 2.947 & 0.044 & 161.9 & 2 \\
\hline PDS 281 & 8.9 & 24 & 1999 Jan 23 & 1.375 & 0.039 & 160.2 & 28 & 0.067 & 0.032 & 106.2 & 1.397 & 0.050 & 161.5 & 1 \\
\hline PDS 286 & 12.2 & 250 & 1999 Jan 18 & 8.217 & 0.060 & 171.8 & 58 & 1.623 & 0.019 & 178.5 & 6.649 & 0.063 & 170.1 & 1 \\
\hline PDS 290 & 14.5 & 300 & 1999 Apr 11 & 2.408 & 0.227 & 149.8 & 234 & 0.669 & 0.012 & 147.8 & 1.741 & 0.227 & 150.6 & 1 \\
\hline GSC 8593-2802 & 12.0 & 300 & 1999 Jan 22 & 1.946 & 0.058 & 115.8 & 448 & 1.146 & 0.007 & 132.8 & 1.184 & 0.058 & 99.4 & 1 \\
\hline HD 85567 & 8.6 & 14 & 1999 Jan 21 & 0.478 & 0.035 & 105.7 & 174 & 0.715 & 0.035 & 116.1 & 0.317 & 0.049 & 42.2 & 2 \\
\hline PDS 303 & 9.3 & 25 & 1999 Jan 21 & 0.579 & 0.044 & 125.3 & 273 & 0.587 & 0.018 & 116.8 & 0.173 & 0.048 & 167.3 & 1 \\
\hline PDS 037 & 13.5 & 300 & 1999 Jan 18 & 3.253 & 0.104 & 120.1 & 217 & 0.530 & 0.010 & 131.9 & 2.775 & 0.104 & 117.9 & 1 \\
\hline PDS 315 & 10.9 & 80 & 1999 Jan 21 & 2.141 & 0.034 & 158.6 & 681 & 0.931 & 0.007 & 173.3 & 1.406 & 0.035 & 149.1 & 1 \\
\hline GSC 8618-2363 & 12.0 & 300 & 1999 Apr 8 & 1.493 & 0.070 & 64.9 & 352 & 0.703 & 0.004 & 128.5 & 1.998 & 0.070 & 56.8 & 1 \\
\hline HD 94509 & 9.1 & 120 & 1999 Apr 8 & 0.688 & 0.015 & 123.1 & 322 & 0.480 & 0.007 & 143.1 & 0.445 & 0.017 & 101.1 & 2 \\
\hline HD 95881 & 8.3 & 10 & 1999 Jan 21 & 1.504 & 0.034 & 116.2 & 81 & 1.500 & 0.021 & 122.6 & 0.335 & 0.040 & 74.7 & 2 \\
\hline PDS 327 & 8.5 & 12 & 1999 Jan 21 & 0.613 & 0.032 & 114.0 & 276 & 0.428 & 0.019 & 130.4 & 0.343 & 0.037 & 92.7 & 1 \\
\hline HD 97048 & 8.5 & 10 & 1999 Jan 22 & 2.519 & 0.044 & 143.3 & 3 & 3.629 & 0.066 & 138.1 & 1.238 & 0.079 & 37.5 & 2 \\
\hline PDS 339 & 7.8 & 7 & 1999 Jan 21 & 0.056 & 0.040 & 99.9 & 114 & 0.261 & 0.047 & 95.0 & 0.206 & 0.062 & 3.6 & 1 \\
\hline PDS 340 & 6.8 & 3 & 1999 Jan 21 & 0.236 & 0.039 & 50.6 & 24 & 3.204 & 0.127 & 126.0 & 3.412 & 0.133 & 36.9 & 1,2 \\
\hline PDS 057 & 9.2 & 40 & 1999 Jan 21 & 0.737 & 0.027 & 90.9 & 488 & 1.122 & 0.017 & 89.3 & 0.388 & 0.032 & 176.2 & 1,2 \\
\hline PDS 344 & 13.2 & 300 & 1999 Apr 11 & 1.590 & 0.053 & 56.4 & 304 & 1.681 & 0.019 & 89.4 & 1.783 & 0.056 & 26.7 & 1 \\
\hline PDS 061 & 6.6 & 2 & 1999 Jan 22 & 0.032 & 0.063 & 167.2 & 20 & 1.699 & 0.086 & 116.7 & 1.705 & 0.107 & 26.1 & 1,2 \\
\hline PDS 140 & 13.1 & 300 & 1999 Apr 11 & 1.852 & 0.099 & 89.4 & 476 & 0.773 & 0.008 & 83.6 & 1.106 & 0.099 & 93.4 & 1 \\
\hline PDS 353 & 13.2 & 480 & 2000 Jun 21 & 0.666 & 0.056 & 22.5 & 1034 & 1.733 & 0.004 & 84.1 & 2.170 & 0.056 & 1.5 & 1 \\
\hline Hen 3-847 & 10.6 & 80 & 1999 Apr 11 & 0.348 & 0.035 & 20.7 & 186 & 0.631 & 0.017 & 53.0 & 0.575 & 0.039 & 159.6 & 2 \\
\hline PDS 361 & 12.9 & 600 & 2000 Jun 22 & 0.219 & 0.034 & 1.3 & 1109 & 0.398 & 0.004 & 97.5 & 0.614 & 0.034 & 5.3 & 1 \\
\hline PDS 364 & 13.5 & 300 & 1999 Apr 11 & 2.298 & 0.030 & 68.8 & 411 & 2.600 & 0.006 & 70.3 & 0.327 & 0.031 & 171.1 & 1 \\
\hline PDS 067 & 13.5 & 600 & 2000 Jun 21 & 0.768 & 0.067 & 7.4 & 666 & 2.287 & 0.004 & 66.3 & 2.731 & 0.067 & 163.5 & 1 \\
\hline PDS 069 & 9.8 & 60 & 1999 Feb 12 & 0.681 & 0.040 & 147.0 & 22 & 0.245 & 0.055 & 112.9 & 0.632 & 0.068 & 157.5 & 1 \\
\hline HD 130437 & 10.0 & 120 & 1999 Apr 10 & 5.818 & 0.085 & 56.9 & 20 & 1.252 & 0.034 & 61.6 & 4.587 & 0.092 & 55.6 & 2 \\
\hline HBC 596 & 12.8 & 300 & 1999 Apr 11 & 4.198 & 0.181 & 46.8 & 115 & 1.482 & 0.015 & 59.8 & 2.939 & 0.182 & 40.4 & 2 \\
\hline
\end{tabular}


Table 1

(Continued)

\begin{tabular}{|c|c|c|c|c|c|c|c|c|c|c|c|c|c|c|}
\hline \multirow[t]{2}{*}{ Object } & \multirow{2}{*}{$\begin{array}{c}V^{\mathrm{a}} \\
(\mathrm{mag})\end{array}$} & \multirow{2}{*}{$\begin{array}{c}\text { Exposure } \\
\text { (s) }\end{array}$} & \multirow[t]{2}{*}{ UT Date } & \multicolumn{3}{|c|}{ Observed } & \multicolumn{4}{|c|}{ Foreground } & \multicolumn{3}{|c|}{ Intrinsic } & \multirow[t]{2}{*}{ Ref. $^{\text {b }}$} \\
\hline & & & & $\begin{array}{c}P \\
(\%) \\
\end{array}$ & $\begin{array}{l}\sigma_{P} \\
(\%)\end{array}$ & $\begin{array}{l}\text { P.A. } \\
\text { (deg) }\end{array}$ & $\begin{array}{l}\text { No. of } \\
\text { Objects }\end{array}$ & $\begin{array}{c}P \\
(\%)\end{array}$ & $\begin{array}{c}\sigma_{P} \\
(\%)\end{array}$ & $\begin{array}{l}\text { P.A. } \\
\text { (deg) }\end{array}$ & $\begin{array}{c}P \\
(\%)\end{array}$ & $\begin{array}{l}\sigma_{P} \\
(\%)\end{array}$ & $\begin{array}{l}\text { P.A. } \\
\text { (deg) }\end{array}$ & \\
\hline HD 132947 & 8.9 & 15 & 1999 Apr 7 & 1.254 & 0.042 & 56.1 & 62 & 1.293 & 0.016 & 59.9 & 0.173 & 0.045 & 6.5 & 2 \\
\hline PDS 389 & 14.2 & 300 & 1999 Jul 28 & 4.630 & 0.218 & 133.4 & 54 & 0.708 & 0.018 & 56.4 & 5.275 & 0.219 & 135.0 & 1 \\
\hline PDS 394 & 13.5 & 300 & 1999 Jul 27 & 2.235 & 0.166 & 15.0 & 312 & 1.448 & 0.010 & 50.9 & 2.252 & 0.166 & 176.1 & 1 \\
\hline PDS 395 & 8.4 & 10 & 1999 Apr 7 & 0.062 & 0.110 & 147.6 & 100 & 1.755 & 0.099 & 43.1 & 1.808 & 0.148 & 133.7 & 1 \\
\hline PDS 144 & 12.8 & 300 & 1999 Apr 10 & 4.653 & 0.070 & 124.1 & 108 & 0.759 & 0.013 & 116.0 & 3.930 & 0.071 & 125.6 & 1 \\
\hline PDS 398 & 7.1 & 3 & 1999 Nov 7 & 0.680 & 0.027 & 86.7 & 9 & 2.665 & 0.090 & 82.4 & 1.995 & 0.094 & 170.9 & 1,2 \\
\hline PDS 399 & 8.6 & 15 & 1999 Apr 10 & 1.705 & 0.022 & 61.0 & 385 & 1.553 & 0.018 & 54.9 & 0.378 & 0.028 & 91.2 & 1 \\
\hline PDS 076 & 8.7 & 10 & 1999 Apr 7 & 0.803 & 0.039 & 81.5 & 20 & 0.077 & 0.027 & 85.6 & 0.727 & 0.047 & 81.1 & 1,2 \\
\hline PDS 406 & 13.9 & 300 & 1999 Apr 10 & 4.719 & 0.081 & 33.6 & 348 & 2.049 & 0.009 & 17.1 & 3.201 & 0.081 & 43.8 & 1 \\
\hline PDS 078 & 8.2 & 10 & 1999 Apr 7 & 0.367 & 0.059 & 14.5 & 65 & 0.455 & 0.047 & 16.4 & 0.092 & 0.075 & 114.1 & 1,2 \\
\hline HD 144668 & 7.0 & 6 & 1999 Apr 8 & 0.579 & 0.019 & 166.5 & 7 & 0.498 & 0.010 & 5.6 & 0.361 & 0.021 & 137.2 & 2 \\
\hline PDS 080 & 9.1 & 14 & 1999 Apr 7 & 0.039 & 0.052 & 45.8 & 47 & 0.589 & 0.059 & 4.9 & 0.585 & 0.079 & 93.0 & 1 \\
\hline PDS 415 & 12.0 & 200 & 1999 Apr 8 & 1.418 & 0.022 & 28.5 & 59 & 1.536 & 0.019 & 29.7 & 0.133 & 0.029 & 132.9 & 1 \\
\hline Hen 3-1191 & 13.7 & 300 & 1999 Apr 10 & 5.885 & 0.146 & 46.0 & 546 & 0.752 & 0.006 & 43.0 & 5.138 & 0.146 & 46.4 & 2 \\
\hline HD 150193 & 8.9 & 15 & 1999 Apr 7 & 4.780 & 0.108 & 56.7 & 34 & 4.330 & 0.057 & 56.1 & 0.460 & 0.122 & 62.4 & 2 \\
\hline PDS 431 & 13.4 & 300 & 1999 Jul 28 & 1.276 & 0.175 & 38.4 & 222 & 1.086 & 0.007 & 35.3 & 0.229 & 0.175 & 53.8 & 1 \\
\hline V921 Sco & 11.4 & 80 & 1999 Apr 7 & 2.509 & 0.256 & 114.7 & 133 & 1.343 & 0.016 & 121.7 & 1.249 & 0.256 & 107.2 & 2 \\
\hline KK Oph & $11.9^{\mathrm{d}}$ & 45 & 1999 Apr 10 & 3.426 & 0.092 & 169.6 & 62 & 0.913 & 0.063 & 33.3 & 3.505 & 0.112 & 162.1 & 2 \\
\hline PDS 453 & 12.9 & 200 & 1999 Apr 7 & 3.608 & 0.060 & 48.9 & 412 & 2.635 & 0.021 & 7.3 & 4.208 & 0.064 & 68.1 & 1 \\
\hline PDS 095 & 11.0 & 100 & 1999 Apr 11 & 1.634 & 0.096 & 34.3 & 70 & 1.041 & 0.022 & 165.6 & 2.048 & 0.098 & 49.4 & 1 \\
\hline PDS 096 & 11.0 & 100 & 1999 Apr 11 & 1.662 & 0.031 & 175.3 & 95 & 1.329 & 0.011 & 177.5 & 0.352 & 0.033 & 166.9 & 1 \\
\hline $\operatorname{PDS} 465^{\mathrm{e}}$ & 12.9 & 300 & 1999 Apr 11 & 9.152 & 0.251 & 43.4 & 501 & 1.298 & 0.009 & 48.3 & 7.876 & 0.251 & 42.6 & 1 \\
\hline PDS 469 & 12.8 & 300 & 1999 Apr 11 & 1.315 & 0.031 & 72.4 & 637 & 1.739 & 0.007 & 62.7 & 0.663 & 0.032 & 132.1 & 1 \\
\hline PDS 473 & 6.9 & 4 & 1999 Apr 8 & 0.417 & 0.035 & 36.0 & 63 & 1.140 & 0.063 & 42.5 & 0.740 & 0.072 & 136.1 & 1,2 \\
\hline PDS 477 & 14.4 & 300 & 1999 Jul 28 & 1.188 & 0.090 & 24.8 & 196 & 0.900 & 0.012 & 66.1 & 1.395 & 0.091 & 4.9 & 1 \\
\hline PDS 514 & 8.2 & 12 & 1999 Apr 8 & 0.095 & 0.029 & 105.2 & 125 & 0.892 & 0.024 & 100.6 & 0.798 & 0.038 & 10.1 & 1 \\
\hline PDS 518 & 12.2 & 300 & 1999 Jul 28 & 1.816 & 0.040 & 93.6 & 4 & 3.051 & 0.089 & 43.7 & 3.807 & 0.098 & 119.6 & 1,2 \\
\hline VV Ser & $11.6^{\mathrm{d}}$ & 400 & 2000 Jun 21 & 1.780 & 0.043 & 77.5 & 5 & 1.566 & 0.079 & 54.5 & 1.322 & 0.090 & 106.7 & 2 \\
\hline MWC 300 & 10.5 & 300 & 2000 Jun 21 & 4.843 & 0.035 & 58.2 & 265 & 4.001 & 0.012 & 55.2 & 0.960 & 0.037 & 71.1 & 2 \\
\hline PDS 520 & 14.7 & 300 & 1999 Jul 28 & 3.513 & 0.107 & 15.2 & 9 & 0.391 & 0.029 & 70.1 & 3.664 & 0.111 & 12.3 & 1 \\
\hline HBC 284/1 & 12.5 & 360 & 2000 Jun 22 & 0.916 & 0.095 & 67.1 & 501 & 0.637 & 0.009 & 56.8 & 0.390 & 0.095 & 84.6 & 2 \\
\hline HBC $284 / 2$ & 12.5 & 360 & 2000 Jun 22 & 0.691 & 0.114 & 72.6 & 501 & 0.637 & 0.009 & 56.8 & 0.365 & 0.114 & 105.6 & 2 \\
\hline PDS 530 & 14.0 & 300 & 1999 Jul 28 & 12.305 & 0.650 & 55.4 & 186 & 1.262 & 0.019 & 50.8 & 11.061 & 0.650 & 55.9 & 1 \\
\hline PDS 543 & 12.5 & 360 & 2000 Jun 21 & 1.105 & 0.025 & 136.8 & 35 & 0.610 & 0.029 & 175.4 & 1.138 & 0.038 & 121.0 & 1 \\
\hline PDS 545 & 8.8 & 18 & 1999 Apr 8 & 3.036 & 0.025 & 78.6 & 44 & 1.129 & 0.036 & 74.5 & 1.925 & 0.044 & 81.0 & 1 \\
\hline PDS 551 & 16.6 & 600 & 2000 Jun 22 & 10.930 & 0.629 & 117.7 & 30 & 0.468 & 0.029 & 69.2 & 10.997 & 0.630 & 118.9 & 1 \\
\hline PDS 564 & 7.4 & 4 & 1999 Apr 8 & 0.481 & 0.034 & 103.7 & 43 & 0.471 & 0.080 & 4.4 & 0.939 & 0.087 & 99.1 & 1,2 \\
\hline WW Vul & 10.5 & 100 & 2000 Jun 21 & 0.849 & 0.070 & 151.3 & 336 & 2.065 & 0.011 & 12.2 & 2.118 & 0.071 & 113.9 & 2 \\
\hline PDS $581^{\mathrm{e}}$ & 11.7 & 80 & 1999 Apr 7 & 12.427 & 0.145 & 44.4 & 772 & 0.326 & 0.008 & 69.5 & 12.221 & 0.145 & 43.8 & 1 \\
\hline HD 190073 & 7.8 & 10 & 1998 Nov 25 & 0.377 & 0.026 & 94.5 & 15 & 0.439 & 0.054 & 97.9 & 0.079 & 0.060 & 25.2 & 2 \\
\hline
\end{tabular}

Notes.

${ }^{\text {a }}$ Magnitudes are from Torres (1999) or SIMBAD.

b The references of the last column are: (1) Vieira et al. 2003; (2) Thé et al. 1994; (3) Torres 1999.

${ }^{c}$ The images of the field containing HD23302 and HD23480 do not include objects with signal high enough to estimate the foreground polarization.

d $B$-band magnitude.

e PDS465 and PDS581 are post-AGB objects.

(This table is also available in a machine-readable form in the online journal.)

the scattering of the central source emission off the circumstellar material. In the path from the object to the observer, the ISM introduces a foreground polarization. These two polarizations are combined vectorially to produce the observed polarization. Hence, to obtain the intrinsic polarization, one must estimate the foreground value to be subtracted from the observed polarization.

The striking majority of stars in the sky are either intrinsically unpolarized or have small, uncorrelated intrinsic polarization. The stellar field is hence dominated by objects presenting only the interstellar component. For our purposes, we may then estimate the foreground polarization toward the object of interest by a weighted average of the polarization of the field stars with a good signal-to-noise ratio ( $\mathrm{S} / \mathrm{N} ; P>3 \sigma_{P}$ ). The average was done from the Stokes parameters $Q$ and $U$. The number of objects in each of our fields vary from 3 to more than 1000. Such a high number of field objects having angular distances to the $\mathrm{HAeBe}$ smaller than $5^{\prime}$ gives us confidence that this technique to estimate the interstellar component is reliable. It is also probably statistically better than the estimates in previous works in which the foreground objects are located angularly farther from the YSO and in smaller numbers due to the use of a photomultiplier as the detector.

The foreground and intrinsic polarizations, as well as the number of field objects used in the estimate of the interstellar polarization, are shown in Table 1 . The polarization of each 


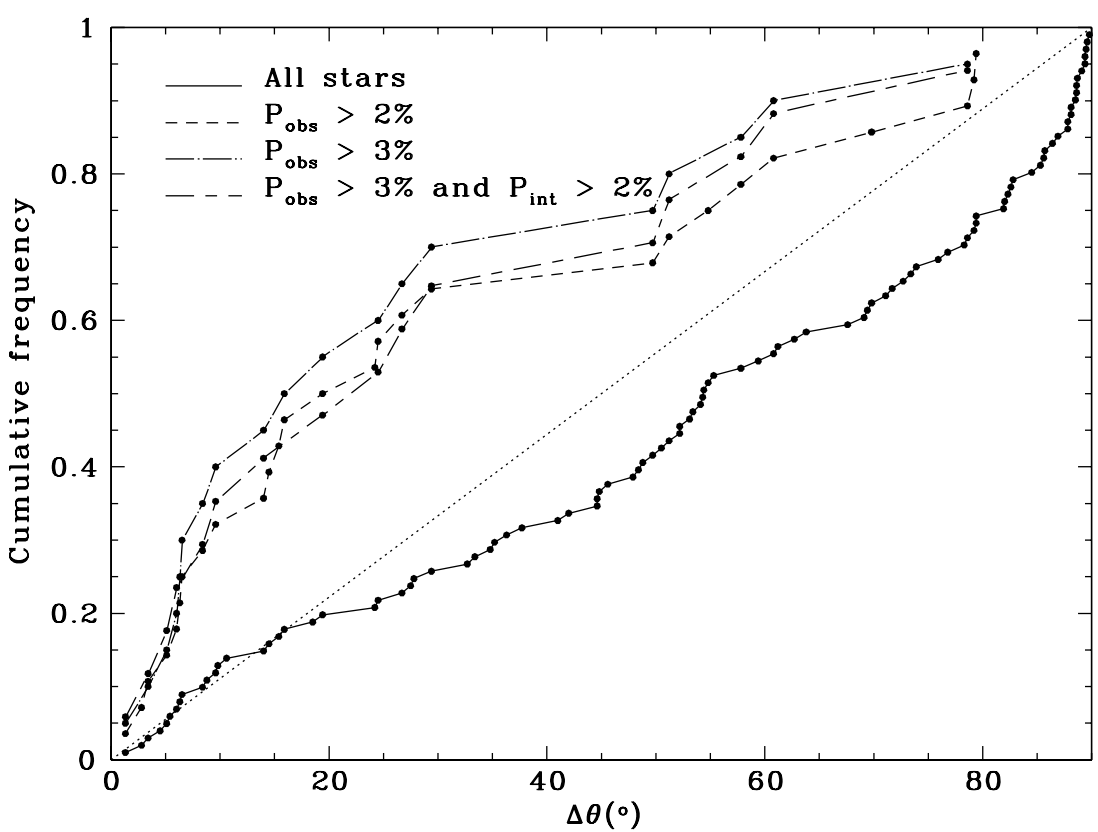

Figure 1. Cumulative frequency distributions of the difference between the intrinsic and interstellar polarization angle, $\Delta \theta$, for our HAeBe sample and subsamples. See the text for details.

foreground star for each field will be available as a Vizier catalog as well as the polarization vectors superposed on an optical image. Two objects, HD 23302 and HD 23480, are too bright and there is no other object in the image with sufficient signal to noise to enable an estimate to the foreground polarization. These two objects are not included in the following analysis. Our final sample analysis is therefore composed by 100 objects.

\section{RESULTS AND DISCUSSION}

The interstellar polarization is caused by aligned aspherical grains that produce the dichroism of the ISM. The alignment mechanism is not yet completely understood. The classical mechanism is based on paramagnetic dissipation by rotating grains with superparamagnetic inclusions (Davis \& Greenstein 1951; Jones \& Spitzer 1967). However, this mechanism may not be efficient enough. Recently, a promising mechanism based on radiative torques has been proposed (Lazarian \& Hoang 2007). In both cases, however, the direction of the magnetic field projected in the plane of the sky can be traced by the position angle of the optical interstellar polarization. A recent review on grain alignment can be found in Lazarian (2007).

The intrinsic polarization position angle is related to the axis of symmetry of the HAeBe envelope. It depends on many factors, but we can say in a simple way that in an optically thin envelope the polarization is parallel to the symmetry axis, and in an optically thick case it is perpendicular. A proper understanding of this issue is obtained by the modeling of the scattering of the central source light in the YSO circumstellar material (e.g., Brown \& McLean 1977; Bastien \& Menard 1990; Whitney \& Hartmann 1993).

Is the geometry of $\mathrm{HAeBe}$ stars in our sample related to the direction of the interstellar magnetic field? We try to answer such a question by checking whether the position angles of the intrinsic polarization and the surrounding interstellar polarization are correlated. For this purpose, we define $\Delta \theta$ as the difference between the intrinsic and foreground polarization directions. $\Delta \theta$ runs from 0 to $90^{\circ}$.
Figure 1 (solid line) shows the cumulative histogram of $\Delta \theta$ for the 100 objects of our sample. The dotted straight line represents the behavior of a uniform distribution. To compare quantitatively both distributions, we used the Kuiper statistic (e.g., Paltani 2004). It is a modification of the Kolmogorov-Smirnov test and is appropriate for cyclic quantities such as $\Delta \theta$. Figure 1 shows that the sample as a whole is obviously not uniform. Indeed, the resulting Kuiper statistic has a probability of only $1.3 \%$, so the hypothesis that our observed distribution is uniform can be discarded. In addition, as the observed curve stays below that of the uniform distribution, it means that $\Delta \theta$ concentrates around $90^{\circ}$.

An inspection of the data shows that this behavior is caused by objects with small values of observed polarization. In this case, the intrinsic polarization has the same modulus as the foreground polarization but is perpendicular to it; this causes the concentration of $\Delta \theta$ near $90^{\circ}$. This probably arises as a result of two factors. The HAeBe object may be nearer to us than most of the field stars. In that case, the foreground polarization must be negligible and the observed polarization should not have been corrected by a foreground component. In addition, if the real intrinsic polarization is undetected given the errors, our estimate of the intrinsic polarization is wrong: it simply reflects the foreground polarization rotated by $90^{\circ}$. We may add that a situation in which the interstellar polarization would be perpendicular and have the same modulus of the intrinsic component is less likely. As a result of this discussion, the concentration of $\Delta \theta$ around $90^{\circ}$ may carry an observational bias.

To circumvent such problems, we also built the cumulative histogram considering only objects having the observed polarization larger than 3\% (Figure 1, dot-dashed line)-these are 19 in number. These objects are less affected by the foreground correction, since they must have a larger contribution from the intrinsic polarization to the observed value. In addition, the polarization in all these cases has a high $\mathrm{S} / \mathrm{N}$ and hence a well determined interstellar component. In this sample, the Kuiper statistic for $\Delta \theta$ has a probability smaller than $2 \%$. Using (the 
27) objects with polarization larger than $2 \%$ (Figure 1, dashed line), the statistic has this same probability. We also note that, as shown in Figure 1, these two samples now present $\Delta \theta$ clustered around zero.

Star-forming regions are dense portions of the ISM. Consequently, they usually present high values of interstellar extinction and polarization. Because of that, we have also checked whether the above results might be biased by cases in which the observed polarization is dominated by the interstellar component. Figure 1 (short dash-long dash line) shows the cumulative distribution considering the objects with the observed polarization larger than $3 \%$ and the intrinsic polarization larger than $2 \%$ (16 objects), a subsample that excludes cases where the interstellar component is the predominant one. This constraint does not modify significantly the histogram and the resulting Kuiper statistic has a probability of $12 \%$.

We concluded that the observed distribution of $\Delta \theta$ for the subsample of objects presenting high signal-to-noise measurements and reliable values of intrinsic polarization is nonuniform with a clear excess of objects with $\Delta \theta$ around zero. This result suggests that the polarization of HAeBe stars has a tendency to be aligned with the ambient interstellar magnetic field. It can be interpreted as an indication that the magnetic field of the material that collapsed to form the star can play a role in defining the YSO geometry and/or the symmetry axis of the envelope.

Our findings are in contrast with the work of Ménard \& Duchêne (2004), which does not show an alignment between the YSO axis and the interstellar magnetic field using a sample of T Tauri stars. However, previous works, using samples of less evolved objects, indicate an alignment (Kobayashi et al. 1978; Dyck \& Lonsdale 1979; Heckert \& Zeilik 1981; Hodapp 1984; Cohen et al. 1984; Strom \& Strom 1987). A possible solution for this discrepancy is that the alignment may be more easily traced in the less evolved, low-mass YSO. This occurs because, during its slow pre-main-sequence evolution, an object can move away from its birth place or present a rotation of its axis direction. From a point of view of the YSO mass, HAeBe stars evolve faster than $\mathrm{T}$ Tauri stars, which could make the observation of the alignment more probable in the higher mass group. We note that in our sample objects with P greater than 3\% also have larger mid-infrared excess (M. J. Sartori et al. 2009, in preparation), putting these objects in an early evolutionary stage.

Some observational arguments have been recently put forward in favor of a fossil origin of the magnetic fields in Ap/ Bp stars (e.g., Wade et al. 2009). This hypothesis assumes that the interstellar magnetic field present in the cloud that originates the star is amplified along the star evolution and is present in the main-sequence stage. This is another piece of evidence that the formed (proto)stellar object can have a memory of the interstellar magnetic field of the parent cloud and its direction, as indicated by our results.

\section{CONCLUSIONS}

We present the results of optical CCD polarimetry of a sample of 102 fields containing $\mathrm{HAeBe}$ stars. The direction of the intrinsic polarization of the YSO, and hence their envelope axis, shows a correlation with interstellar magnetic field direction for the sample as a whole. This result may be an observational bias, as discussed in the text. Subsamples of the more polarized objects present a statistically significant tendency to have the YSO polarization aligned with the interstellar magnetic field. This indicates that the geometry of HAeBe objects retained a memory of the interstellar magnetic field.

We acknowledge the use of NASA's Astrophysics Data System Service and the SIMBAD database, operated at CDS, Strasbourg, France. This work was partially supported by Fapesp (CVR and AMM: Proc. 2001/12589-1; JGH: Procs. 2001/ 09018-2 and 2005/00397-1) and CNPq (AMM).

Facilities: LNA:BC0.6m

\section{REFERENCES}

Bastien, P., \& Menard, F. 1990, ApJ, 364, 232

Brown, J. C., \& McLean, I. S. 1977, A\&A, 57, 141

Cohen, R. J., Rowland, P. R., \& Blair, M. M. 1984, MNRAS, 210, 425

Davis, L., \& Greenstein, J. L. 1951, ApJ, 114, 206

Dyck, H. M., \& Lonsdale, C. J. 1979, AJ, 84, 1339

Gregorio-Hetem, J., Lepine, J. R. D., Quast, G. R., Torres, C. A. O., \& de La Reza, R. 1992, AJ, 103, 549

Heckert, P. A., \& Zeilik, M., II. 1981, AJ, 86, 1076

Hodapp, K.-W. 1984, A\&A, 141, 255

Jones, R. V., \& Spitzer, L. J. 1967, ApJ, 147, 943

Kobayashi, Y., Kawara, K., Maihara, T., Okuda, H., Sato, S., \& Noguchi, K. 1978, PASJ, 30, 377

Lazarian, A. 2007, J. Quant. Spectrosc. Radiat. Transfer, 106, 225

Lazarian, A., \& Hoang, T. 2007, MNRAS, 378, 910

Magalhães, A. M., Rodrigues, C. V., Margoniner, V. E., Pereyra, A., \& Heathcote, S. 1996, in ASP Conf. Ser. 97, Polarimetry of the Interstellar Medium, ed. W. G. Roberge \& D. C. B. Whittet (San Francisco, CA: ASP), 118

McKee, C. F., \& Ostriker, E. C. 2007, ARA\&A, 45, 565

Ménard, F., \& Duchêne, G. 2004, A\&A, 425, 973

Mouschovias, T. C., \& Ciolek, G. E. 1999, in NATO ASIC Proc. 540, The Origin of Stars and Planetary Systems, ed. C. J. Lada \& N. D. Kylafis (Dordrecht: Kluwer), 305

Paltani, S. 2004, A\&A, 420, 789

Pereyra, A. 2000, PhD thesis, Univ. of São Paulo

Pereyra, A., \& Magalhães, A. M. 2002, ApJS, 141, 469

Strom, S. E., \& Strom, K. M. 1987, in Proc. Symp. 115, Star Forming Regions, ed. M. Peimbert \& J. Jugaku (Dordrecht: Reidel), 255

Tamura, M., \& Sato, S. 1989, AJ, 98, 1368

Thé, P. S., de Winter, D., \& Perez, M. R. 1994, A\&AS, 104, 315

Torres, C. A. O. 1999, Special Publ. 10 (Rio de Janeiro Observatório Nacional)

Torres, C. A. O., Quast, G., de La Reza, R., Gregorio-Hetem, J., \& Lépine, J. R. D. 1995, AJ, 109, 2146

Vieira, S. L. A., Corradi, W. J. B., Alencar, S. H. P., Mendes, L. T. S., Torres, C. A. O., Quast, G. R., Guimarães, M. M., \& da Silva, L. 2003, AJ, 126, 2971

Wade, G. A., Alecian, E., Grunhut, J., Catala, C., Bagnulo, S., Folsom, C. P., \& Landstreet, J. D. 2009, arXiv:0901.0347

Whitney, B. A., \& Hartmann, L. 1993, ApJ, 402, 605 